\title{
Tidal Power Extraction on a Streamwise Bed Slope
}

\author{
Aidan Wimshurst, Richard Willden \\ Department of Engineering Science, University of Oxford, Parks Road, OX1 3PJ, UK
}

\begin{abstract}
Three dimensional Reynolds-averaged Navier-Stokes computations are presented for an actuator disc representation of an ideal tidal stream energy extracting device, operating within a channel that slopes in the streamwise direction. Downwards facing, horizontal and upwards facing slopes are considered at the same mass flow rate and depth at the device position, for both high and low channel blockage ratios. The downwards facing slope is shown to present a greater available kinetic power to the disc than the horizontal and upwards facing slopes. This is due to the velocity profile being more strongly sheared as a result of the adverse pressure gradient, which results in greater thrust variation over the disc area. Conversely, during uphill flows devices experience reduced thrust variation, as the velocity profile is more uniform due to the favourable pressure gradient. Although downhill flows can deliver greater overall power due to increased flow shear, uphill flows are more efficient at converting the power presented to the disc into power removed and this is shown to be due to the downstream flow constriction and resulting increased pressure coefficient drop through the bypass flow.
\end{abstract}

Keywords: Bathymetry, Tidal Turbines, OpenFOAM, $k-\omega$ SST, Actuator Disc, Boundary Layer Separation.

\section{Introduction}

Large bathymetric features of the order of the rotor diameter $D$ (such as bed ridges, troughs and hills) induce local changes in the velocity bound5 ary layer, which may lead to considerable unsteady loading on tidal devices if they are not sited optimally. An improved understanding of the influence of such large bathymetric features may be used to better advise the placement of future tidal devices, which are currently positioned based on the local depth, bed condition and predicted mean velocity profile alone. To this end, several authors have used computational fluid dynamics (CFD) with high fidelity bathymetry data and acoustic doppler current profilers (ADCP), to predict the local flow field at prospective tidal sites. Zangiabadi et al. (2015) used large eddy simulation (LES) and steady state Reynolds-averaged Navier-Stokes (RANS) computations to study the local flow field of a poten-

\footnotetext{
*Corresponding author

Email address: Aidan.Wimshurst@eng.ox.ac.uk (Aidan Wimshurst)

Preprint submitted to Ocean Engineering

20 tial tidal stream installation site in Ramsey Sound, Wales. They observed the wake structure and vortices shed from Horse Rock (a submerged pinnacle) and obtained good agreement with ADCP transects of the local flow field. Evans et al. (2015) studied 25 the same site in Ramsey Sound and suggested that the wake recovery behind the submerged pinnacle was influenced both by the velocity magnitude in the flow direction and the local bathymetry. Soto and Escauriaza (2015) computed the local flow field

so through the Chacao channel in Chile, using LES and an actuator disc representation for an array of tidal energy devices. They found that even small bed features led to significant changes in the velocity boundary layer upstream of the discs, adversely 35 affecting the performance of the entire array. When validating a high fidelity CFD simulation of the Minas Passage in Canada, Wilcox et al. (2015) identified a volcanic planform (bed ridge) that lead to a large separation bubble, similarly to flow over 40 a backwards facing step. The high levels of turbulence generated from the volcanic planform and from a nearby island were subsequently highlighted 


\begin{tabular}{|c|c|c|c|}
\hline \multicolumn{4}{|c|}{ Nomenclature } \\
\hline$B$ & Blockage ratio (-) & $\Delta p$ & Static pressure drop across the \\
\hline$\Delta B$ & Log-law intercept & & $\operatorname{disc}\left(\mathrm{N} / \mathrm{m}^{2}\right)$ \\
\hline & modification (-) & $R$ & Diffuser corner radius (m) \\
\hline$c_{f}$ & Skin friction coefficient $(-)$ & $\Delta r$ & Radial cell length \\
\hline$C_{p}$ & Power coefficient (-) & & at the disc edge $(\mathrm{m})$ \\
\hline$C_{p}^{\prime}$ & Alternative power coefficient (-) & $s$ & Device tip to tip spacing $(\mathrm{m})$ \\
\hline$C_{\text {pre }}$ & Static pressure coefficient (-) & $\delta T$ & Local thrust increment $(\mathrm{N})$ \\
\hline$\Delta C_{\text {pre }}$ & $\begin{array}{l}\text { Static pressure coefficient } \\
\text { drop across the disc }(-)\end{array}$ & $U$ & $\begin{array}{l}\text { Reynolds-averaged velocity } \\
\text { vector }(\mathrm{m} / \mathrm{s})\end{array}$ \\
\hline$C_{s}$ & Roughness constant (-) & $U_{b}$ & Bulk velocity $(\mathrm{m} / \mathrm{s})$ \\
\hline$C_{T}$ & Thrust coefficient (-) & $U_{d}$ & Velocity normal to the disc \\
\hline$C_{T}^{\prime}$ & Alternative thrust coefficient (-) & & plane $(\mathrm{m} / \mathrm{s})$ \\
\hline$D$ & Device or disc diameter $(\mathrm{m})$ & $U_{d 0}$ & Velocity normal to the disc \\
\hline E & Empirical constant (-) & & plane with no device $(\mathrm{m} / \mathrm{s})$ \\
\hline$\Delta \dot{E}$ & Change in total energy flux (W) & $u_{\tau}$ & Friction velocity $(\mathrm{m} / \mathrm{s})$ \\
\hline$g$ & $\begin{array}{l}\text { Acceleration due to } \\
\text { gravity }\left(\mathrm{m}^{2} / \mathrm{s}^{2}\right)\end{array}$ & $\overline{u^{2}}, \overline{w^{2}}, \overline{u w}$ & $\begin{array}{l}\text { Reynolds-stress components } \\
\text { in the } x-z \text { plane }\left(\mathrm{m}^{2} / \mathrm{s}^{2}\right)\end{array}$ \\
\hline$G$ & $\begin{array}{l}\text { Cell growth ratio normal to } \\
\text { the wall (-) }\end{array}$ & $U, V, W$ & $\begin{array}{l}\text { Reynolds-averaged velocity } \\
\text { components }(\mathrm{m} / \mathrm{s})\end{array}$ \\
\hline$h$ & Local fluid depth (m) & $x, y, z$ & Cartesian coordinates $(\mathrm{m})$ \\
\hline$\Delta h$ & $\begin{array}{l}\text { Change in local free surface } \\
\text { height }(\mathrm{m})\end{array}$ & $\Delta x$ & $\begin{array}{l}\text { Streawmise cell length } \\
\text { at the disc plane }(\mathrm{m})\end{array}$ \\
\hline$H$ & $\begin{array}{l}\text { Diffuser inlet height or fluid } \\
\text { depth at the disc plane }(\mathrm{m})\end{array}$ & $y^{+}$ & $\begin{array}{l}\text { Non-dimensional wall normal } \\
\text { distance (-) }\end{array}$ \\
\hline$k$ & $\begin{array}{l}\text { Turbulent kinetic energy } \\
\left(\mathrm{m}^{2} / \mathrm{s}^{2}\right)\end{array}$ & $z_{0}$ & $\begin{array}{l}\text { Wall adjacent cell centroid } \\
\text { height }(\mathrm{m})\end{array}$ \\
\hline$K$ & Momentum loss factor (-) & $\epsilon$ & Dissipation rate $\left(\mathrm{m}^{2} / \mathrm{s}^{3}\right)$ \\
\hline$K_{s}$ & Roughness height (m) & $\kappa$ & Empirical constant (-) \\
\hline$K_{s}^{+}$ & Dimensionless roughness & $\nu$ & Kinematic viscosity $\left(\mathrm{m}^{2} / \mathrm{s}\right)$ \\
\hline & height (-) & $\nu_{t}$ & Kinematic eddy viscosity $\left(\mathrm{m}^{2} / \mathrm{s}\right)$ \\
\hline$\dot{m}$ & Mass flow rate $(\mathrm{kg} / \mathrm{s})$ & $\rho$ & Fluid density $\left(\mathrm{kg} / \mathrm{m}^{3}\right)$ \\
\hline$\hat{n}$ & Unit normal vector (-) & $\theta$ & Slope angle $\left({ }^{\circ}\right)$ \\
\hline$p$ & Static pressure $\left(\mathrm{N} / \mathrm{m}^{2}\right)$ & $\tau_{w}$ & Wall shear stress $\left(\mathrm{N} / \mathrm{m}^{2}\right)$ \\
\hline$p_{\text {ref }}$ & Reference static pressure $\left(\mathrm{N} / \mathrm{m}^{2}\right)$ & $\omega$ & Specific dissipation rate $\left(s^{-1}\right)$ \\
\hline
\end{tabular}

as areas to be avoided for device installation, in order to mitigate unnecessary fatigue damage.

The high computational cost associated with sufficiently resolving real bathymetric features both spatially and temporally has led some authors to analyse simplified bathymetric features instead. Draper et al. (2013) and Edmunds et al. (2014) both independently considered an idealised tidal headland site and the effect of tidal energy extraction on the local undisturbed flow field. The results of these studies highlighted that given the local bathymetry of a potential tidal stream energy in55 stallation site, improved device performance could potentially be achieved with careful choice of device location, operating point and local blockage. In addition, changes to the local flow field due to the presence of the devices may adversely effect so the rates of sediment transport, volume flow rate through the channel and lead to other environmental consequences, if not properly considered. 
In this study, the interaction of a simplified general bathymetric feature with an ideal energy ex65 tracting device (representing an axial flow tidal turbine) will be considered. The observations made in this work may be useful when selecting potential tidal stream energy sites and identifying key flow features, before high fidelity CFD studies or ADCP 70 measurements are performed. As shown in Fig. 1] a first approximation to local bathymetric changes can be made by a constant (positive or negative) slope in the streamwise direction. Slope variations in the cross stream (lateral) direction (as may oc75 cur near a coastal headland (Draper et al., 2013)), can be approximated in a similar manner and may be considered in future work. This study presents results for streamwise bed slopes only.

In this investigation a series of computational so studies were performed, to investigate the steady flow changes induced by such a general bed feature. Unsteady flow features such as vortex shedding (induced by bed hills, pinnacles and sharp headlands), were not considered. It should be noted that inviscid, analytical models (such as those of Garrett and Cummins (2007), Houlsby et al. (2008) and Whelan et al. (2009) ) cannot be applied directly to the current problem. This is primarily because the surface pressure distribution acting on the inclined bed 115

90 surface cannot be readily determined and hence the momentum balance remains unclosed. However, the analytical solution for a similar tidal channel with zero bed slope (using the model of Garrett and Cummins (2007), Houlsby et al. (2008) and Whelan 120 et al. (2009)), can still be used to provide a useful reference point and will be presented alongside the computational results in this investigation.

Before presenting the results for idealised tidal turbines, a validation study was performed. This ${ }^{125}$ was to ensure that the turbulence and wall modelling approaches were sufficient to predict the wall shear stress variation (and hence the developing velocity boundary layer) on the inclined bed slope. In particular, accurate modelling was essential for the 130

105 downwards facing slope, which was susceptible to flow separation due to the adverse pressure gradient.

\section{Turbulence Model Validation Study}

Obi et al. (1993) investigated the separation and subsequent reattachment of fully developed turbulent flow from the smooth surface of a nominally two-dimensional asymmetric diffuser. This study 140

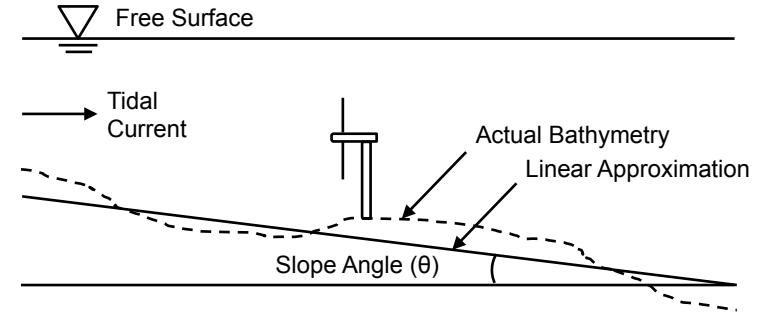

Figure 1: An approximation of large bathymetric change, of the order of the device diameter (such as a bed ridge) by a constant slope angle $\theta$.

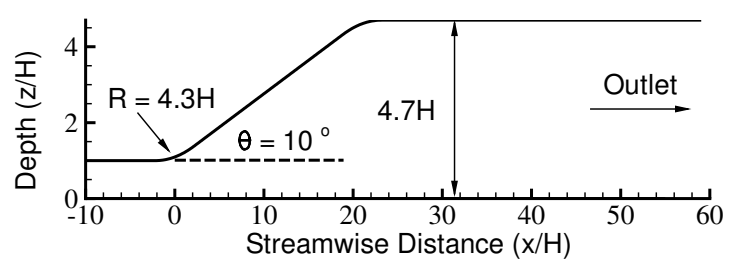

Figure 2: Schematic diagram of the two-dimensional working section of the diffuser for the validation case study. The flow enters at $x / H=-10$ and exits at $x / H=60$.

was chosen as a test case for several reasons. Firstly, it had been adopted as a benchmark test in the IAHR/ERCOFTAC workshop to assess the performance of turbulence models (Hellsten and Rautaheimo, 1999). Secondly, the measurements were independently verified with a repeat experiment by Buice and Eaton (1997) using hot wire probes. Their measurements of the core flow variables were in excellent agreement with the data set of Obi et al. (1993). In addition, they added skin friction $\left(\tau_{w}\right)$ measurements (for the inclined wall) to the data set and extended the region of mean flow measurements further downstream. Skin friction measurements were essential to determine the separation point.

The nominally two-dimensional diffuser geometry is shown schematically in the $x-z$ plane in Fig. 2. It consisted of a diffusing section of length $21 \mathrm{H}$, where $H=20 \mathrm{~mm}$ is the height of the upstream inlet section. Downstream of the diffusing section, the geometry extended a further $39 H$ to the exit plane, whilst the inlet was placed $10 H$ upstream of 135 the diffusing section. In the experiments, the diffuser had a width in the $y-z$ plane of $35 H$ at the throat and $7.45 \mathrm{H}$ at the outlet. Therefore following Apsley and Leschziner (2000), the diffuser was assumed to be two-dimensional and was assigned unit width in the $y-z$ plane. Both corners were 
rounded with a corner radius of $4.3 H$, to avoid premature separation at a sharp corner. The slope of the diffuser was $10^{\circ}$.

To ensure fully developed inflow conditions in the 195 computational model, the inlet plane was extended $115 \mathrm{H}$ upstream of the diffuser throat (not shown in Fig. 2 for clarity). Based on the bulk velocity $U_{b}=15.4336 \mathrm{~m} / \mathrm{s}$ (volume flow rate per unit cross sectional area) of the upstream flow, the kine- 200 matic viscosity of air $=1.456 \times 10^{-5} \mathrm{~m}^{2} / \mathrm{s}$ and inlet channel height $H=20 \mathrm{~mm}$, the channel Reynolds number was 21200 . In the original dataset, experimental measurements of the mean flow velocities $U, W$ and Reynolds stresses $\overline{u^{2}}, \overline{w^{2}}, \overline{u w}$ were taken 205 155 between $x / H=3.2$ and $x / H=25.2$ using two component laser-Doppler anemometry (LDA). Surface pressure measurements were taken from the plane wall using pressure taps.

Individual tidal turbines are unlikely to be in- 210 160 stalled on $10^{\circ}$ bed slopes, $5^{\circ}$ being the practical limit for device installation (E.ON, 2014). An expansion ratio of 4.7 for the water depth in the streamwise direction also seems unlikely for real tidal channels. Hence, the test case can instead 215 165 by viewed as an upper limit for the range of applicability of the turbulence and wall modelling approaches. It should be emphasised that the purpose of the validation study was to investigate the performance of the turbulence models under the influence 220 170 of an adverse pressure gradient and not to replicate a tidal channel.

\subsection{Numerical Method}

The incompressible Reynolds Averaged Navier- 225 Stokes (RANS) equations were solved for the mean

175 flow variables, with the Reynolds Stress tensor modelled using a linear eddy viscosity model, the Boussinesq hypothesis (Pope, 2000). To achieve closure of the RANS equations, the kinematic eddy 230 viscosity $\nu_{t}$ was calculated with the $k-\omega$ shear 180 stress transport (SST) model proposed by Menter (1994). In this model, $k$ represents the turbulent kinetic energy and $\omega$ the specific dissipation rate. The updated empirical model constants and blend- 235 ing functions proposed by Menter et al. (2003) were adopted, rather than those in the original $k-\omega \mathrm{SST}$ model (Menter, 1994).

In the validation study, comparison was also made with the Wilcox (1988) $k-\omega$ model and the 240 low Reynolds number implementation of the $k-\epsilon$ model (with wall damping functions), proposed by Launder and Sharma (1974). For these turbulence closure schemes, the original model constants and damping functions were adopted.

At the inlet plane a fixed value of 0 was applied for the turbulent scalars ( $k$ and $\omega$ ), a uniform profile of $15.4336 \mathrm{~m} / \mathrm{s}$ was applied for the velocity $(\boldsymbol{U})$ and a zero normal gradient boundary condition for the pressure $(p)$. At the domain outlet, zero gradient conditions were applied for the velocity and turbulent scalars, whilst the pressure was assigned a fixed value of 0 . In addition, for both the plane and the inclined wall, a fixed value boundary condition $k=0$ was applied for the turbulent kinetic energy. For the specific dissipation rate $\omega$, the asymptotic solution for the viscous sub layer was applied, following Menter (1994).

The wall adjacent cell height was set such that the cell centroid was well within the viscous sublayer $\left(y^{+}=u_{\tau} y / \nu<1\right.$ where $u_{\tau}=\sqrt{\tau_{w} / \rho}, \nu$ is the kinematic viscosity and $\rho$ is the fluid density) along the entire plane and inclined surfaces, with a cell wall normal growth ratio of 1.05. A mesh sensitivity study (not shown here for brevity) revealed less than a $1 \%$ change in the computed separation point relative to the length of the diffusing section $(21 H)$, with a further reduction in $y^{+}$to $<0.5$. The original mesh was therefore deemed sufficiently resolved for the present investigation.

Whilst flow separation is inherently 3D and unsteady, a steady state solution to the 2D RANS equations was sought. This was for consistency with the results of similar RANS computations presented by Apsley and Leschziner (2000).

The open source code OpenFOAM (version 2.3.1) was adopted for the computations. OpenFOAM employs a collocated, cell centred, finite volume approach for all flow variables, with a Rhie-Chow type interpolation procedure to prevent pressure oscillations (Rhie and Chow, 1983). The momentum equations and the Poisson equation for the pressure correction were solved in terms of their primitive variables and the SIMPLE algorithm (Patankar. 1980) was employed for the pressure velocity coupling. Central differencing was applied for face interpolation of the Laplacian and gradient terms, whilst linear upwind differencing was applied for the convection terms.

The evolution of the flow field in the expanding section of the diffuser was monitored during the solution process using point probes. Convergence to a steady state was deemed sufficient when the integrated surface pressure and skin friction forces remained within $1 \%$ of their final value over 100 iter- 


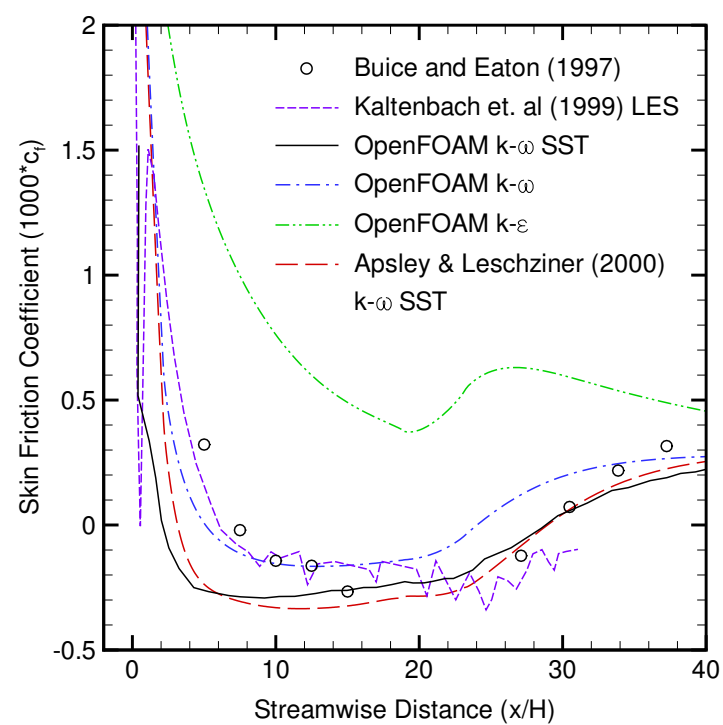

Figure 3: Comparison of the predicted skin friction variation over the inclined surface of the diffuser for various turbulence models, with experimental measurements.

ations. In addition the residual of all primitive flow variables (velocity, pressure and turbulence scalars) normalised by their initial values, was required to be reduced to below $10^{-6}$.

\subsection{Results}

Experimental measurements based on the wall hear stress indicated that the flow separated from the inclined surface of the diffuser wall at $x / H \approx$ 7.5. Subsequent reattachment occurred downstream of the throat at $x / H \approx 29$ (Obi et al., 1993). To compare the measured and calculated separation wall skin friction coefficient $c_{f}=\tau_{w} / \frac{1}{2} \rho U_{b}^{2}$.

The closest agreement with the experimental measurements of the separation point, for the OpenFOAM RANS models, was provided by the

${ }_{260}$ Wilcox $k-\omega$ model at $x / H \approx 5$. However, the reat- 310 tachment point was under predicted at $x / H \approx 24$. The low Reynolds number Launder and Sharma variation of the $k-\epsilon$ model (with wall damping functions), over predicted the wall shear stress at all locations on the inclined surface of the diffuser 315 wall. Therefore the flow did not separate from the inclined surface at all. Such results with the $k-\epsilon$ model have also been reported in the literature $\mathrm{Ap}$ sley and Leschziner, 2000).

The $k-\omega$ SST turbulence model predicted the 320 reattachment point in good agreement with the ex- perimental data at $x / H \approx 29$ but predicted the separation point too early, at $x / H \approx 3$. These findings were in good agreement with the $k-\omega$ SST 275 computations performed by Apsley and Leschziner (2000), that are also shown in Fig. 3

To overcome the limitations of the 2D steady state RANS approach, Kaltenbach et al. (1999) presented computations using 3D LES. Their results are also included in Fig. 3 for comparison. The time averaged LES results showed the separation point at $x / H \approx 6$, in much closer agreement with the experimental measurements of Buice and Eaton (1997). In addition, they also showed a point of lo285 cal separation at the throat of the diffuser, due to a periodic shear layer instability. However, this could not be verified by experimental measurement and was not predicted by any of the RANS models presented by Apsley and Leschziner (2000).

The influence of the predicted separation and reattachment points on the mean velocity is demonstrated in Fig. 4a. For the $k-\epsilon$ model the predicted velocity profile appeared almost symmetric, whilst the $k-\omega$ SST model and the experimental measurements showed a definite asymmetry. Although it provided a better prediction of the separation point, the $k-\omega$ model did not capture the mean velocity profile as well as the $k-\omega$ SST model. For tidal turbine simulations, the rotor is typically located at mid depth of the water column. This region of the velocity profile was well predicted by the $k-\omega$ SST model, despite its premature separation point.

From Fig. 4p, it was clear that the $k-\omega$ SST model predicted the Reynolds shear stress profiles closest to the experimental measurements. However, it was clear that none of the linear eddy viscosity models considered captured these profiles faithfully. Such limitations have also been reported by Apsley and Leschziner (2000). Non-linear eddy viscosity models (NLEVM) or differential Reynolds stress models (DRSM) may be utilised to overcome these limitations. A key conclusion drawn by Apsley and Leschziner was that NLEVM and DRSM did not universally outperform the linear eddy viscosity models, when applied to flow conditions remote from their original calibration. Such an in depth investigation was not the purpose of this study. Therefore, the $k-\omega$ SST model was taken forward due to its comparatively superior prediction of the mean flow quantities, despite its limitations in Reynolds stress prediction. 

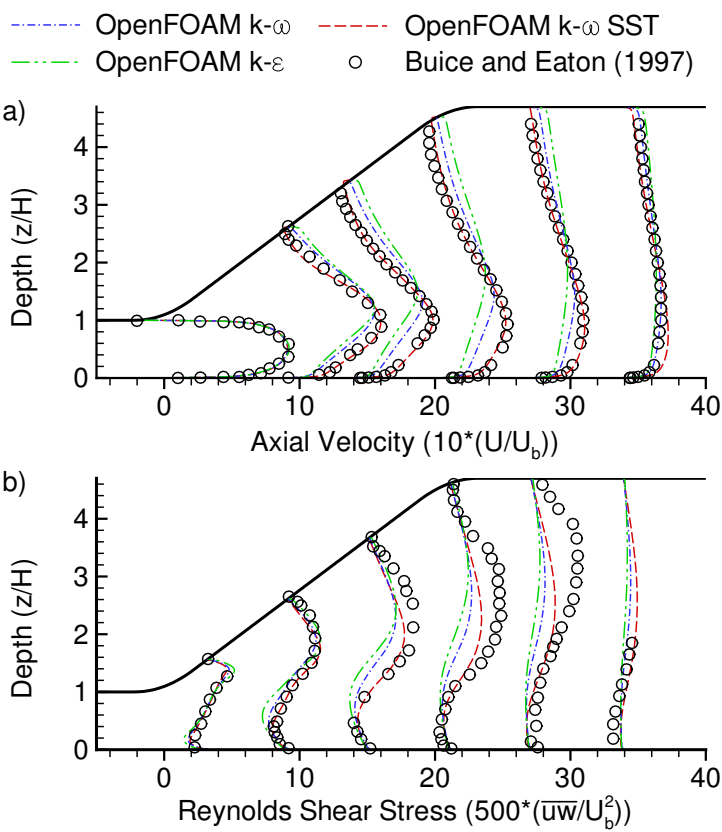

Figure 4: Development of a) the axial velocity profile and b) the Reynolds shear stress using linear eddy viscosity models in OpenFOAM. Following Kaltenbach et al. (1999) and Apsley and Leschziner (2000) each of the profiles has been shifted by $x / H$ on the horizontal axis, in order to separate the profiles.

\section{Streamwise Bed Slope Investigation}

Having investigated the applicability of the $k-\omega$ SST turbulence model to fully developed turbulent wall bounded flows under the influence of an adverse pressure gradient, a similar methodological approach was adopted for the tidal channel investigation. Some small adaptations were first made to include the influence of an ideal axial flow tidal device on the flow and a new computational domain was created.

\subsection{Computational Domain}

Constant slope angles $(\theta)$ of $5^{\circ}, 0^{\circ}$ and $-5^{\circ}$ were taken as representative downwards, horizontal and upwards facing slopes. Higher slope angles are unlikely to be practical for installation purposes and were therefore not considered (E.ON, 2014). Typical installation depths for tidal turbines are likely to be of the order $40 \mathrm{~m}$, with current designs of turbine with diameter around $20 \mathrm{~m}$, leading to water depths 385 of approximately $2 D$. Installation in shallower water is prohibited due to the need to maintain adequate surface clearance for other users of the chan- nel. For this study, a water depth of $2 D$ at the 345 device plane was adopted for all slope angles and the device centreline was placed at the mid-depth of the channel, $1 D(20 \mathrm{~m})$ below the free surface. To allow direct comparison between the cases, the device was oriented perpendicular to the free surface, 0 ather than the local bed slope.

The constant slope was allowed to propagate $10 \mathrm{D}$ upstream and downstream of the device plane. For a device diameter $D=20 \mathrm{~m}$ and slope angle $\theta=5^{\circ}$, this corresponded to a maximum and minimum waably occur at typical sites). To ensure that the slope was sufficiently far from the inlet and outlet planes, the geometry was then extended horizontally $10 \mathrm{D}$ at both ends. The sharp corners were then rounded to improve numerical stability.

Due to the low channel Froude number of 0.101 (based on the water depth of $40 \mathrm{~m}$ and bulk velocity of $2.0 \mathrm{~m} / \mathrm{s}$ at the device plane) and realistic blockage ratios under consideration here (up to 0.196), free surface deformation effects were neglected by modelling the free surface as a rigid lid. Subsequent calculations were performed to estimate the neglected change in free surface height $\Delta h$, with the device operating at maximum thrust for the high blockage case. To perform this estimation, the total energy flux removed from the flow $\Delta \dot{E}$ was computed numerically as the difference between the total energy flux at the computational domain inlet (subscript 1) and outlet (subscript 2), using equation 1

$$
\begin{aligned}
\Delta \dot{E} & =\int_{0}^{h_{1}}\left(p_{1}+\frac{1}{2} \rho U_{1}^{2}\right) U_{1} d S_{1} \\
& -\int_{0}^{h_{2}}\left(p_{2}+\frac{1}{2} \rho U_{2}^{2}\right) U_{2} d S_{2}
\end{aligned}
$$

$h$ refers to the local fluid depth and $d S$ integration over the channel cross sectional area. By neglecting the energy dissipated to wake mixing downstream of the computational domain outlet, the change in free surface height was then estimated using $\Delta h=\Delta \dot{E} /(\dot{m} g)$. For the device operating at maximum thrust, the expected free surface height change was calculated as $0.24 \%$ of the water depth at the device location. For the computations performed here (at lower device thrust), the free surface deformation was expected to be even smaller and was therefore neglected. Similar computations performed by Consul et al. (2013) also showed that 


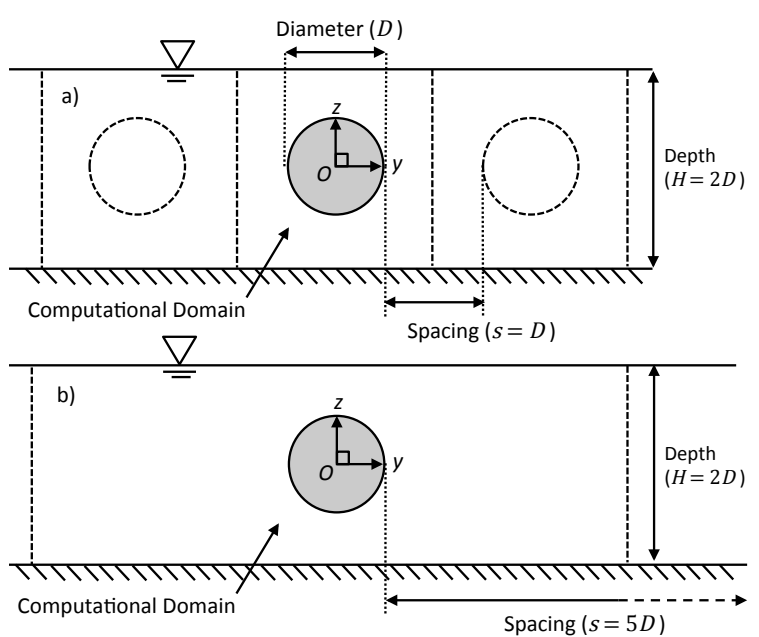

Figure 5: An infinite fence (single line) of devices with tip to tip spacing a) $s=D$ and b) $s=5 D$. The local blockage ratio is a) 0.196 and b) 0.065 .

even for high blockage ratios (up to 0.50) the max-

imum free surface height change was only $0.51 \%$ of the undisturbed upstream free surface height and therefore the rigid lid assumption was deemed to be a reasonable approximation.

In this study the hydrodynamic limit of power extraction was of interest, rather than the performance of a specific device and therefore an actuator disc representation of the device was adopted, fol- 425 lowing Nishino and Willden (2012). The effect of the disc on the flow was modelled as a discontinuous drop in static pressure $(\Delta p)$, applied locally over the disc area using equation 2

$$
\Delta p=K\left(\frac{1}{2} \rho U_{d}^{2}\right) \quad U_{d}=\boldsymbol{U} \cdot \hat{\boldsymbol{n}}
$$

$K$ is the momentum loss factor, a parameter that controls the load or thrust acting on the disc, $\hat{\boldsymbol{n}}$ is the unit vector normal to the disc face and $U_{d}$ the local velocity normal to the disc face. The device was assumed to be located within an infinite fence (a single line) of devices, extending in the lateral direction across the entire channel. As shown in Fig. 5, a representative high blockage case $(B=\pi / 16=0.196)$ with tip to tip spacing $s=D$ and representative low blockage case ${ }_{430}$ $(B=\pi / 48=0.065)$ with tip to tip spacing $s=5 D$, were investigated.

A schematic diagram of the computational do415 main for the downwards facing slope $\theta=5^{\circ}$, is shown in Fig. 6. This choice of geometry was 435

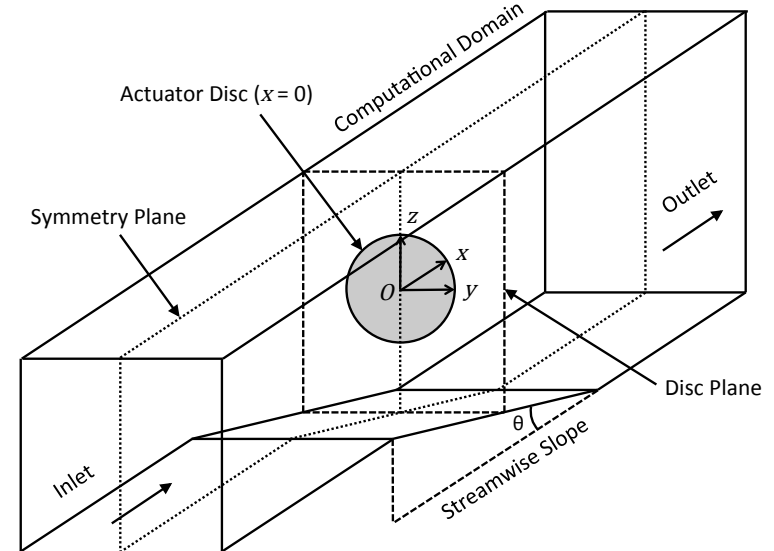

Figure 6: Schematic diagram of the computational domain for the downwards facing slope $\left(\theta=5^{\circ}\right)$, demonstrating the plane of symmetry and Cartesian coordinate system adopted. The flow direction is reversed for the upwards facing slope $\left(\theta=-5^{\circ}\right)$.

adopted to allow both upwards and downwards facing slopes to be simulated using the same computational domain, with the flow direction reversed for the upwards facing slope $\left(\theta=-5^{\circ}\right)$. The plane of symmetry (along the device centreline) was also utilised to reduce the mesh size, improving the computational efficiency.

A range of device operating points were simulated by varying the momentum loss factor $K$ for each bed slope and local blockage. A summary of the parameter space investigated is provided in Table 1.

Table 1: Summary of parameter space investigated

\begin{tabular}{|c|c|c|c|}
\hline$s$ & $B$ & $\theta\left({ }^{o}\right)$ & $K$ \\
\hline \multirow{3}{*}{$D$} & & 5 & $0,1,2,2.5, \ldots 5.5,6.0$ \\
\hline & 0.196 & 0 & $0,1,2,2.5, \ldots 5.5,6.0$ \\
\hline & & -5 & $0,1,2,2.5, \ldots 5.5,6.0$ \\
\hline \multirow{3}{*}{$5 D$} & & 5 & $0,1,2,2.5, \ldots 5.5,6.0$ \\
\hline & 0.065 & 0 & $0,1,2,2.5, \ldots 5.5,6.0$ \\
\hline & & -5 & $0,1,2,2.5, \ldots 5.5,6.0$ \\
\hline
\end{tabular}

\subsection{Mesh Structure}

A high Reynolds number approach was adopted for the bed surface, such that the wall adjacent cell centroid was placed everywhere within the logarithmic law region $\left(y^{+}>30\right)$. It was not possible to place the cells within the viscous sub-layer (strictly $\left.y^{+}<5\right)$ due to high cell aspect ratios. Further 
streamwise and lateral mesh refinement (to reduce the cell aspect ratios sufficiently), was also not attempted as this would have resulted in excessively large meshes. Therefore, the velocity profile between the wall adjacent cell centroid and the wall was modelled using the standard logarithmic law (log law) velocity profile (Pope, 2000), which was assumed to be sufficient, even for the downwards facing slope (with an adverse pressure gradient).

${ }_{445}$ This assumption was expected to provide an acceptable prediction of the wall shear stress, as long as flow separation did not occur.

To ensure that sufficient cells were placed within the boundary layer, the cell growth ratio normal to the wall $(G)$ was investigated. A summary of the parameters adopted for the study is provided in Table 2, The wall adjacent cell centroid height was held constant at $z_{0} / D=1 \times 10^{-4}$, to attain a minimum $y^{+}=34.5$. A further reduction of $z_{0} / D$

455 was not attempted, as the universal law of the wall does not follow a logarithmic velocity profile in the buffer region $\left(5<y^{+}<30\right)$ (Pope, 2000).

\begin{tabular}{ccccc}
\multicolumn{5}{c}{ Table 2: Mesh sensitivity study parameters } \\
\hline$z_{0} / D$ & $G$ & $y_{\min }^{+}$ & $y_{\max }^{+}$ & Cells \\
\hline $1 \times 10^{-4}$ & 1.1 & 34.5 & 85.1 & $1.41 \times 10^{6}$ \\
$1 \times 10^{-4}$ & 1.05 & 34.5 & 85.1 & $1.75 \times 10^{6}$ \\
$1 \times 10^{-4}$ & 1.02 & 34.5 & 85.1 & $2.50 \times 10^{6}$ \\
\hline
\end{tabular}

Fig. 7] shows the variation in skin friction coefficient, for the downwards facing slope $\left(\theta=5^{\circ}\right)$ with no device present $(K=0)$. The downwards facing slope was chosen to test the mesh as it was most susceptible to separation (from the adverse pressure gradient) and hence the skin friction coefficient expected to be most sensitive to cell growth ratio.

It was clear from Fig. 7, that the skin friction coefficient was everywhere positive and hence the flow did not separate on the downwards facing slope. The wall modelling approach was therefore deemed acceptable in this situation. In addition, Fig. 7 480 showed a difference at the point of minimum skin friction of $3.6 \%$ for a growth ratio $G=1.1$ and $3.3 \%$ for a growth ratio $G=1.05$, when compared to the predicted skin friction coefficient for the lowest cell growth ratio $G=1.02$ (finest mesh). There- ${ }^{485}$ fore, a cell growth ratio of 1.1 away from the wall was adopted for future computations, to minimise the total cell count and hence computational load, without introducing substantive error.

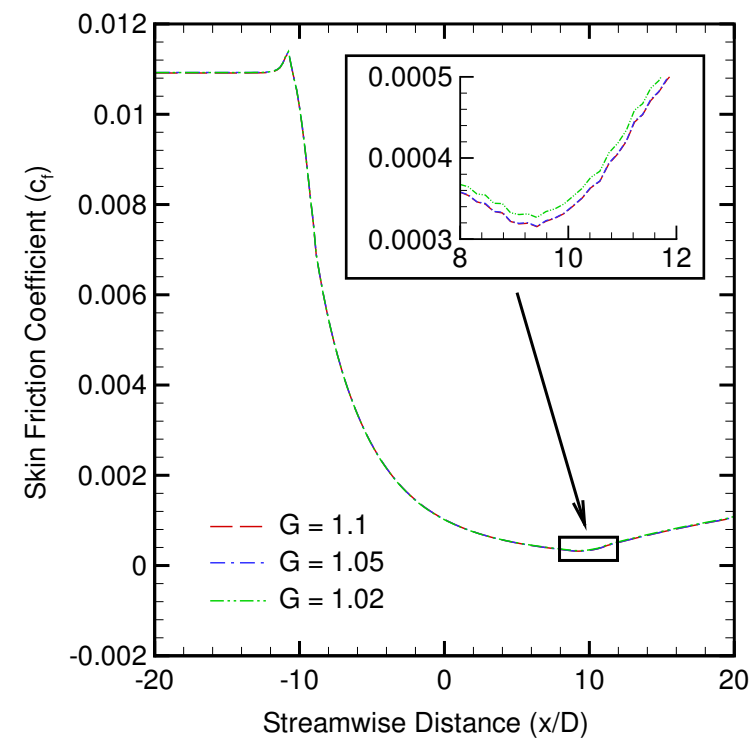

Figure 7: Streamwise variation in skin friction coefficient, for the downwards facing slope $\left(\theta=5^{\circ}\right)$ with no device present $(K=0)$, for various growth ratios $G$.

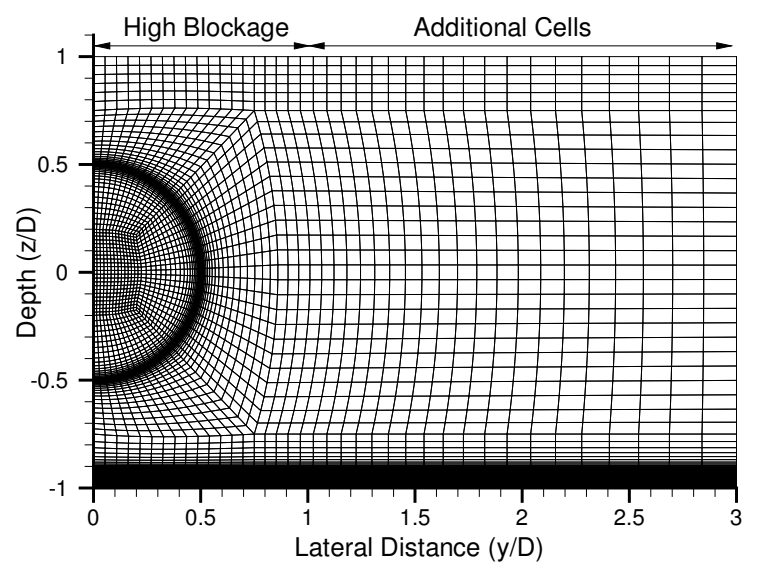

Figure 8: A cut through the block structured mesh at the disc plane $(x / D=0)$, for the low blockage case $(B=0.065)$.

It should be noted that increasing the device thrust (by increasing the momentum loss factor $K$ ) leads to an increase in bypass flow velocity and hence velocity gradient at the wall. Therefore with increasing device thrust, the wall skin friction increased downstream of the device and the onset of flow separation was delayed. The accuracy of the wall modelling approach was therefore not expected to degrade with increasing device thrust.

A cut through the block structured mesh structure at the disc plane is shown in Fig. 8 for the 
low blockage case $(B=0.065)$. For the high blockage case, the additional cells between $y / D=1$ and 3 were removed, thus maintaining the same mesh structure around the actuator disc. The mesh cross section shown in Fig. 8 was extruded in the streamwise $(x)$ direction (away from the disc plane), starting with an initial cell length $\Delta x=D / 200$, with a cell growth ratio of 1.02 . The expansion was continued up to a cell length of $D / 5$, far upstream and downstream of the disc. The mesh resolution at the disc plane (with a disc edge resolution of $\Delta r=D / 400)$, corresponds to the 'medium' mesh case of Nishino and Willden (2012) where it was shown to be sufficient to capture the thin shear layer that develops between the core and bypass flow passages behind the disc.

\subsection{Inlet Conditions}

Fully developed, velocity and turbulence scalar $(k, \omega)$ profiles were applied at the inlet to the computational domain, with the same mass flow rate for all bed slopes. The mass flow rate was chosen to achieve a bulk velocity $U_{b}=2.0 \mathrm{~m} / \mathrm{s}$ at the disc plane (a typical rated flow speed for tidal turbines). Several techniques have been proposed to generate such profiles, which have been reviewed by Tabor and Baba-Ahmadi (2010). In this study, a mapping plane was chosen over a precursor simulation to mitigate interpolation errors. As shown in Fig. 9 , the current solution was sampled at $5 D$ downstream of the inlet (negligible change was found in the final profiles with the location of the sample plane) and the sampled velocity and turbulence scalars profiles applied at the inlet, in the next iteration. As a result, the fully developed, consistent inlet profiles were generated as the steady flow solution converged.

The fully developed inlet profiles were compared with the device operating at zero and maximum thrust and a maximum difference of $0.9 \%$ was observed in the velocity profile. The disturbance created by the device was therefore deemed sufficiently small to be ignored when generating the inlet profiles.

The fully developed profiles at the inlet were functions of the mass flow rate, the local depth 565 and the bed roughness alone. As the mass flow rate and local depth were already specified, the bed roughness height $K_{s}$ was used to control the velocity shear and turbulence intensity. Following Cebeci and Bradshaw (1977), the log law intercept was

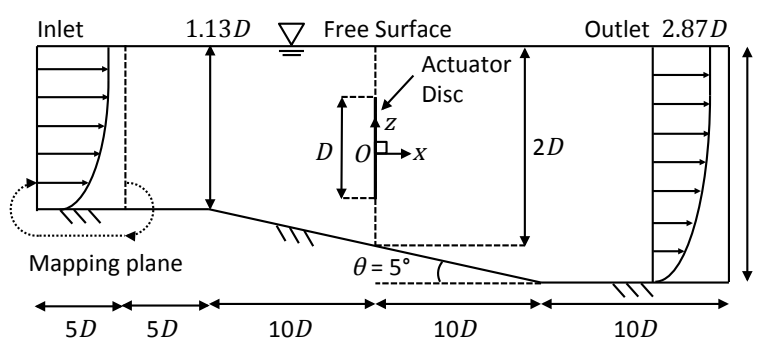

Figure 9: Schematic diagram of a cross-section of the computational domain in the $x-z$ plane, for the downwards facing slope $\left(\theta=5^{\circ}\right)$. The location of the mapping plane for the computational domain inlet $(x / D=-15)$ and other key dimensions are also indicated.

modified by $\Delta B$ to account for the wall roughness, via equations 3 and 4 .

$$
\begin{aligned}
& u^{+}=\frac{1}{\kappa} \ln \left(E y^{+}\right)-\Delta B \\
& \Delta B=\Delta B\left(K_{s}^{+}, C_{s}, E, \kappa\right)
\end{aligned}
$$

$E$ and $\kappa$ are empirical constants (taken as 9.8 and 0.41 respectively, as proposed by Cebeci and Bradshaw (1977)) and $C_{s}$ is the roughness constant (taken as 0.5 here for uniform roughness elements, also following Cebeci and Bradshaw (1977)). Fig. 10 shows the fully developed velocity and turbulence intensity profiles (for the horizontal bed slope), with non dimensional roughness height $K_{s}^{+}=K_{s} u_{\tau} / \nu$, for representative hydrodynamically smooth, transitional and rough surfaces. As shown in Fig. 10, the hydrodynamically fully rough surface experienced the greatest velocity shear and highest turbulence intensity (of up to $6 \%$ at the wall). Therefore this inlet profile was chosen as most representative of real tidal sites. Using this method, it was not possible to achieve greater turbulence intensities with further increase in roughness height. To achieve greater turbulence intensities, an unsteady inlet condition such as that proposed by Gant and Stallard (2008) would be required. Such an approach was not applied here due to the preference of maintaining a steady computation.

\section{Results}

\subsection{Shear Profile Development}

Before examining the response of the actuator disc, changes to the velocity profile due to the bed slope were considered. Fig. 11 compares the axial 

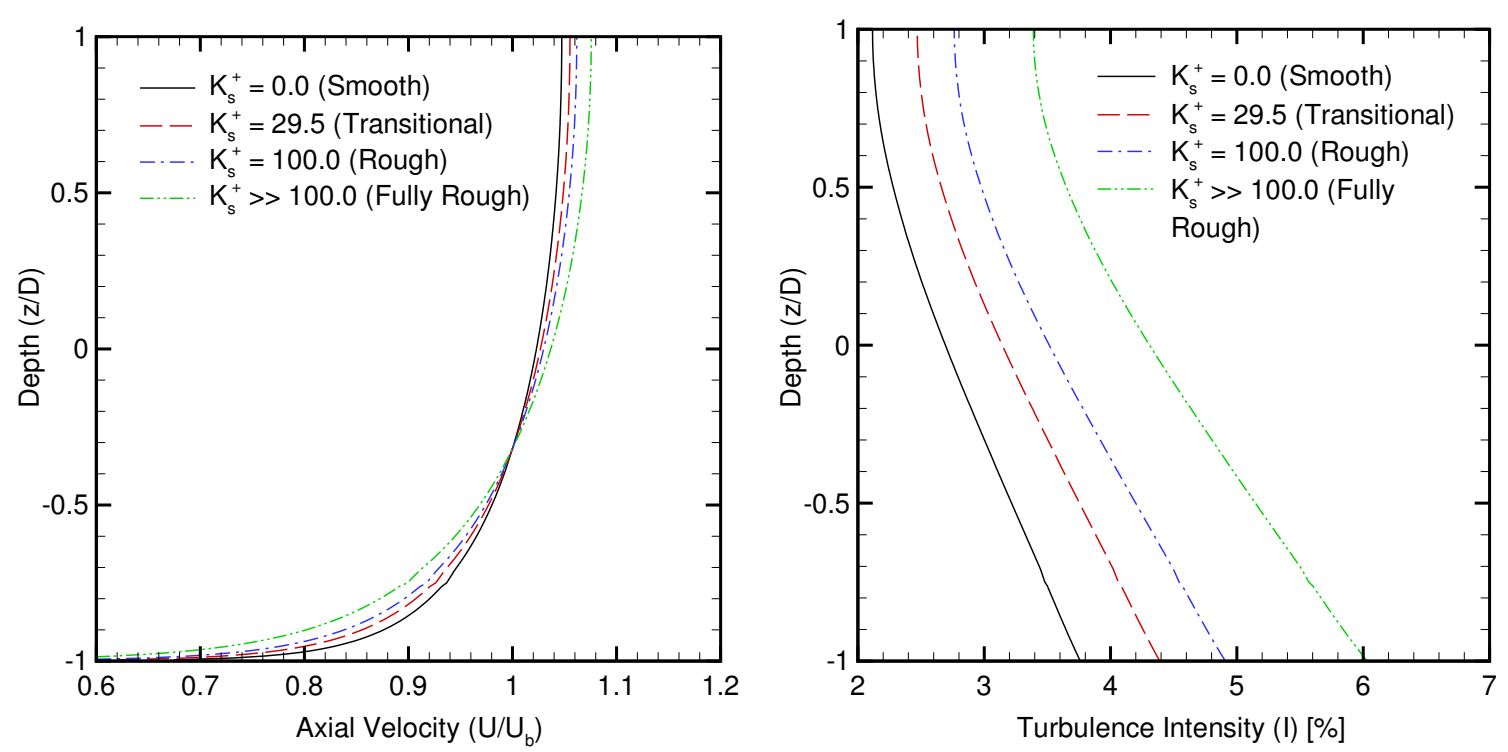

Figure 10: Fully developed axial velocity and turbulence intensity profiles at the computational domain inlet $(x / D=-20)$, for the horizontal bed slope $\left(\theta=0^{\circ}\right)$, for hydrodynamically smooth, transitional and rough bed surfaces.

velocity profiles $(U)$ at the disc plane with no device present $(K=0)$. Recall that at the disc plane, the local depth $H=40 \mathrm{~m}$ and bulk velocity $U_{b}=2.0$ $\mathrm{m} / \mathrm{s}$ were the same for all bed slopes and hence we chose to normalise $U$ by the bulk flow velocity, to allow direct comparison between the profiles. It should also be noted that the axial velocity $U$ shown in Fig 11 is equivalent to $U_{d}$, as the axial velocity is normal to the disc at the disc plane and the disc is perpendicular to the free surface.

For the downwards facing slope, the adverse pressure gradient lead to a reduction in wall shear stress along the bed slope. Consequently, the velocity gradient at the wall reduced and velocity gradient away from the wall increased (in order to maintain a constant mass flux). The velocity profile therefore became highly sheared across the disc face for the downwards facing slope. For the upwards facing slope the opposite occurred, with the favourable pressure gradient reducing the shear across the disc face, leading to a more uniform profile. This reduction in shear for the upwards facing slope is intuitively expected and is often utilised in wind tunnels with a Bernoulli restriction at the inlet.

The developing shear profiles on the streamwise bed slopes significantly affected the total ki- 600 netic power available to the $\operatorname{disc}\left(\int_{A} \frac{1}{2} \rho K U_{d}^{3} d A\right)$ and the total thrust exerted by the disc on the

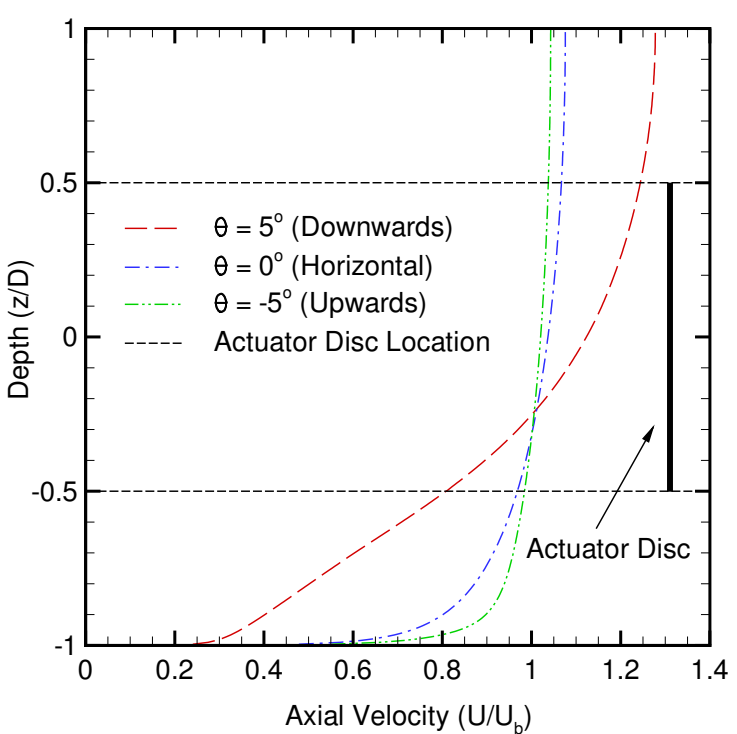

Figure 11: Axial velocity profiles at the disc plane $(x / D=$ $0)$, with no disc present $(K=0)$. The disc height is indicated by dashed horizontal lines.

flow $\left(\int_{A} \frac{1}{2} \rho K U_{d}^{2} d A\right)$. Fig. 12 compares the kinetic power available to the disc and the thrust exerted by the disc on the flow, expressed in terms of an available kinetic power coefficient $\left(C_{p}\right)$ and disc thrust coefficient $\left(C_{T}\right)$. These coefficients were cal- 
culated by dividing the total disc thrust and available kinetic power by a reference thrust and power ased on the bulk velocity $U_{b}$, as shown in equa- ${ }_{650}$ tions 5 and 6 . The angular braces $\langle\ldots\rangle$ have been used to represent the spatial average of the enclosed quantity over the disc area.

$$
\begin{aligned}
& C_{T}=\frac{\int_{A} \frac{1}{2} \rho K U_{d}^{2} d A}{\frac{1}{2} \rho U_{b}^{2} A}=K \frac{\left\langle U_{d}^{2}\right\rangle}{U_{b}^{2}} \\
& C_{p}=\frac{\int_{A} \frac{1}{2} \rho K U_{d}^{3} d A}{\frac{1}{2} \rho U_{b}^{3} A}=K \frac{\left\langle U_{d}^{3}\right\rangle}{U_{b}^{3}}
\end{aligned}
$$

When adopting this normalisation, the reference thrust and power were the same for all bed slopes and hence the available kinetic power coefficient and disc thrust coefficient directly represented the power and thrust available to that disc under those 6 operating conditions. Alternative normalisations based on volumetric averages over the disc plane to account for velocity shear, will be considered later on.

Adopting this definition of available kinetic power coefficient, all 3 bed slopes exceeded the available kinetic power coefficient predicted with the analytic model of Garrett and Cummins (2007), for a horizontal bed slope with zero shear. This is because the local velocity normal to the disc plane cubed and then averaged over the disc area is always greater for sheared profiles, than the equivalent uniform profile with the same mass flux. Fig. 12 also showed that the downwards facing slope had a greater available kinetic power coefficient than the upwards facing and horizontal slopes for all disc hrusts (momentum loss factors) and could achieve a higher peak power coefficient. This increase in available kinetic power coefficient was predicted for both the high and low blockage cases, as the shear profiles developed at the disc plane were due to the bed slope alone.

It should be noted that the increase in available 680 kinetic power coefficient predicted for the actuator disc model considered here may not necessarily translate directly into a greater extractable power output for a real tidal device operating on a downwards facing slope. This is because it may not be ${ }_{685}$ possible to convert the additional available kinetic power from the highly sheared velocity profile to extractable power, without a load mitigation strategy such as cyclical pitch control. Fig. 13 shows the local disc thrust $\delta T=\frac{1}{2} \rho K U_{d}^{2} d A$ acting on an 690 increment of disc area $d A$ divided by the average thrust for that disc, in order to demonstrate the non-uniformity of the thrust exerted by the disc in reaction to the sheared velocity profile. The maximum thrust exerted by the disc was up to $15 \%$ greater and the minimum $30 \%$ lower than the average, for a disc operating on the downwards facing slope. This was due to the significant shear in the 5 velocity profile induced by the downwards facing bed slope. For a real device, the cyclical blade loading (with each rotor revolution) was therefore likely to be more severe on a downwards facing slope.

From a loading perspective it appeared at this 660 stage that an upwards facing slope would be most desirable, in order to achieve a more uniform velocity profile over the swept area of the device, thus leading to a more uniform thrust variation. However, the change in flow passage expansion downstream of the device (due to the bed slope) and its effect on disc thrust and power extraction, has not yet been considered.

\subsection{Flow Passage Expansion}

To examine the effect of flow passage expansion downstream of the disc, the influence of the shear profile required isolating. Hence, an alternative normalisation was adopted, based on the velocity normal to the disc plane (for that bed slope) with no device present $U_{d 0}$, which was then averaged over the disc plane to define a reference velocity $\left\langle U_{d 0}\right\rangle$. A similar normalisation was adopted in order to compute a reference dynamic pressure $\frac{1}{2} \rho\left\langle U_{d 0}^{2}\right\rangle$ and kinetic energy flux $\frac{1}{2} \rho\left\langle U_{d 0}^{3}\right\rangle$, using equations 7 and 8.

$$
\begin{aligned}
& \left\langle U_{d 0}^{2}\right\rangle=\frac{1}{A} \int_{A}(U(K=0) \cdot \hat{\boldsymbol{n}})^{2} d A \\
& \left\langle U_{d 0}^{3}\right\rangle=\frac{1}{A} \int_{A}(U(K=0) \cdot \hat{\boldsymbol{n}})^{3} d A
\end{aligned}
$$

Fig. 14 shows contours of normalised axial velocity downstream of the disc for the high blockage ratio case $(B=0.196)$, at a momentum loss factor $K=5$ (close to the peak power coefficient) for each slope. The dividing streamlines are also shown, to indicate the core and bypass flow passages. 'Bypass' flow here refers to that which passes around the disc and 'core' flow, that which passes through the disc. As shown in Fig. 14, the key effect of the bed slope and the free surface was to provide a restriction on the core flow passage area, downstream of the disc. For the upwards facing slope, the bed 
a) $B=0.196$

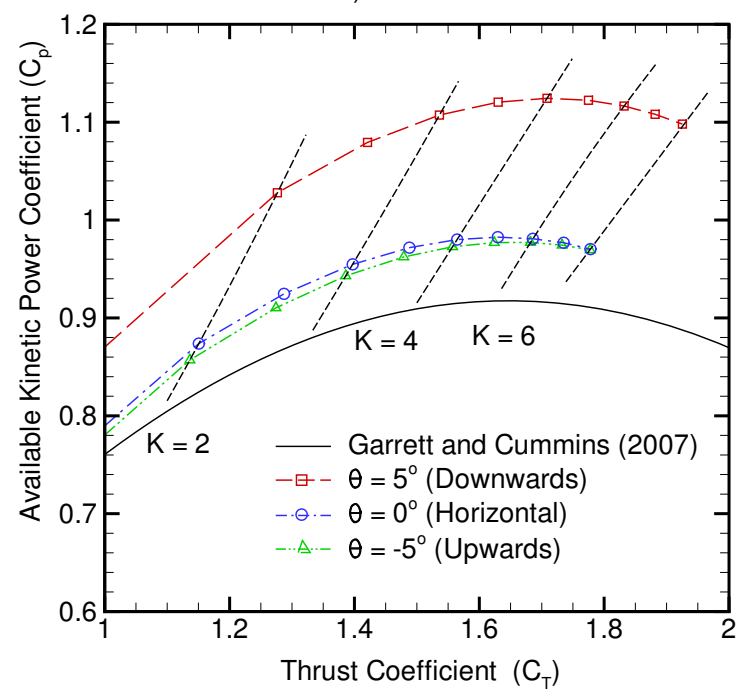

b) $B=0.065$

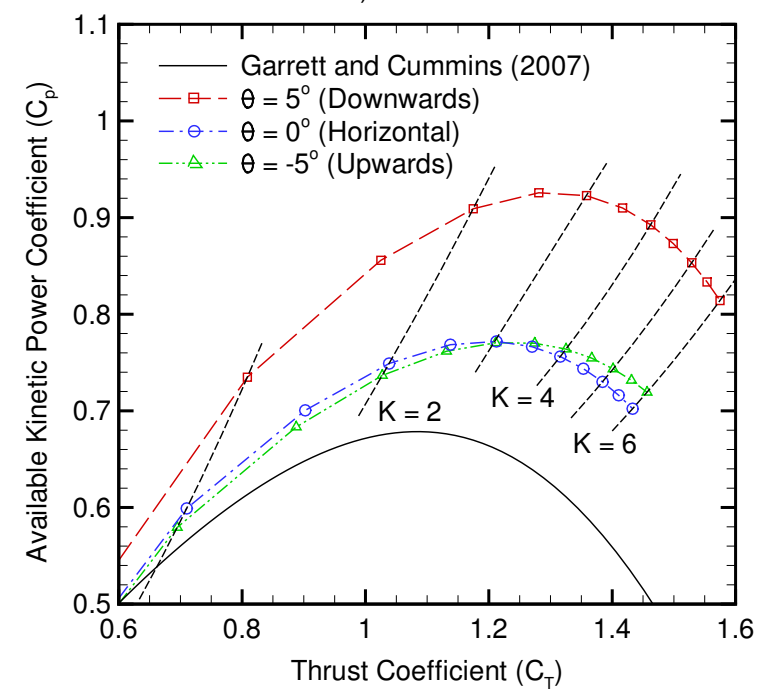

Figure 12: Available kinetic power coefficient variation with disc thrust coefficient for the downwards facing $\left(\theta=5^{\circ}\right)$, horizontal $\left(\theta=0^{\circ}\right)$ and upwards facing slopes $\left(\theta=-5^{\circ}\right)$, for a) the high blockage case $(B=0.196)$ and b) the low blockage case $(B=0.065)$.
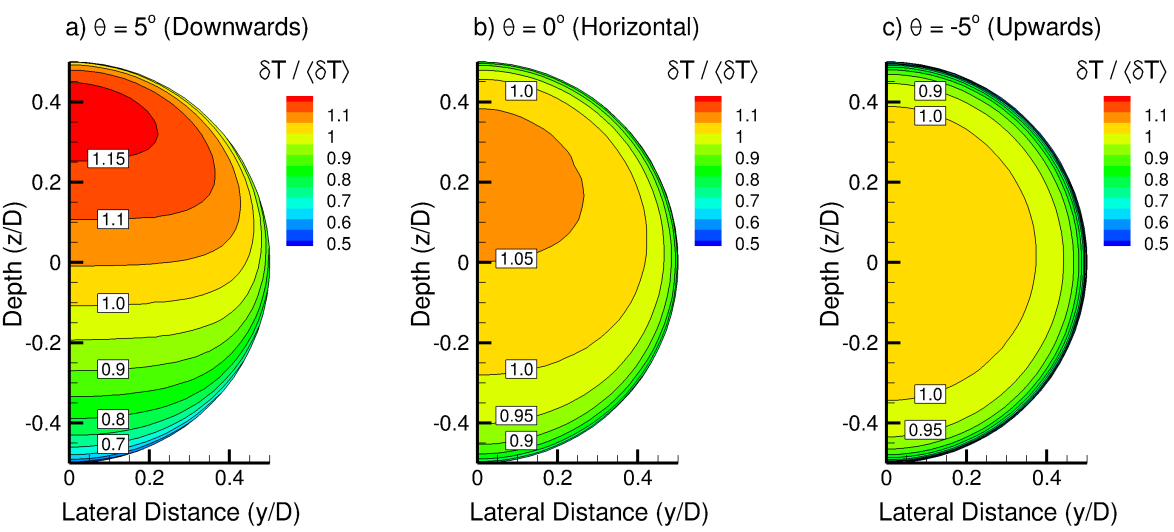

Figure 13: Thrust variation over the disc face at $K=5$ for (a) downwards facing slope $\left(\theta=5^{\circ}\right)(\mathrm{b})$ horizontal slope $\left(\theta=0^{\circ}\right)$ (c) upwards facing slope $\left(\theta=-5^{\circ}\right)$ at a blockage ratio $B=0.196$.

slope and free surface acted to further constrain the expansion of the core flow passage. Whereas for the downwards facing slope, the core flow passage was 705 able to expand further than for the horizontal bed slope. As the mass flow rate was consistent between these cases, the upwards facing slope experienced a reduced velocity deficit in the core flow passage and a greater mean velocity in the bypass flow passages, 710 than the horizontal and downwards facing slopes.

Furthermore, Fig. 15 shows the centreline axial velocity along the local mid-depth of the chan- nel, normalised by the centreline axial velocity with no device present $(K=0)$. Due to the increased shear and resultant increase in mixing between the core and bypass flow passages, the maximum velocity deficit on the upwards facing slope reduced and moved further downstream of the device plane. In contrast, the downwards facing slope experienced an increased maximum velocity deficit, which occurred closer to the device plane.

In addition, Fig. 16 compares the location of the downstream wake edge for the downwards facing, 

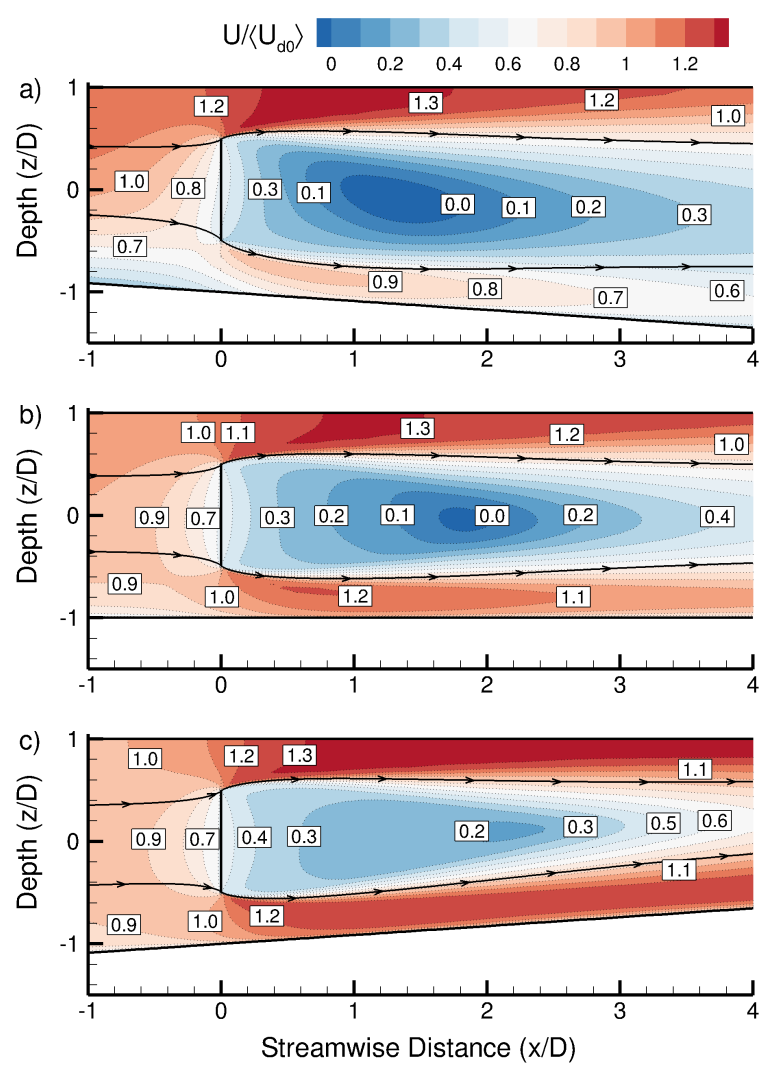

Figure 14: Normalised axial velocity contours for $K=5$ (near the peak of the power coefficient curve) for (a) downwards facing slope $\left(\theta=5^{\circ}\right)(\mathrm{b})$ horizontal slope $\left(\theta=0^{\circ}\right)(\mathrm{c})$ upwards facing slope $\left(\theta=-5^{\circ}\right)$, for the high blockage case $(B=0.196)$.

horizontal and upwards facing slopes, as a result of the flow passage expansion. Following the approach of Masters et al. (2013) in order to account for the developing sheared velocity profile, the wake edge has been defined as the location where the axial velocity reached $95 \%$ of the axial velocity with no device present $(K=0)$. Due to the increased velocity shear between the core and bypass flow passages leading to increased wake mixing, the wake width (taken as the distance between the top and bottom wake edges) reduced more rapidly for the 735 upwards facing slope than for the downwards facing and horizontal slopes. Hence, in combination with the centreline velocity profiles in Fig. 15, it can be deduced that the overall rate of wake recovery increased for the device on the upwards facing slope and reduced for the device on the downwards facing slope.

The additional restriction of the flow passages observed here (due to the bed slope), is concep-

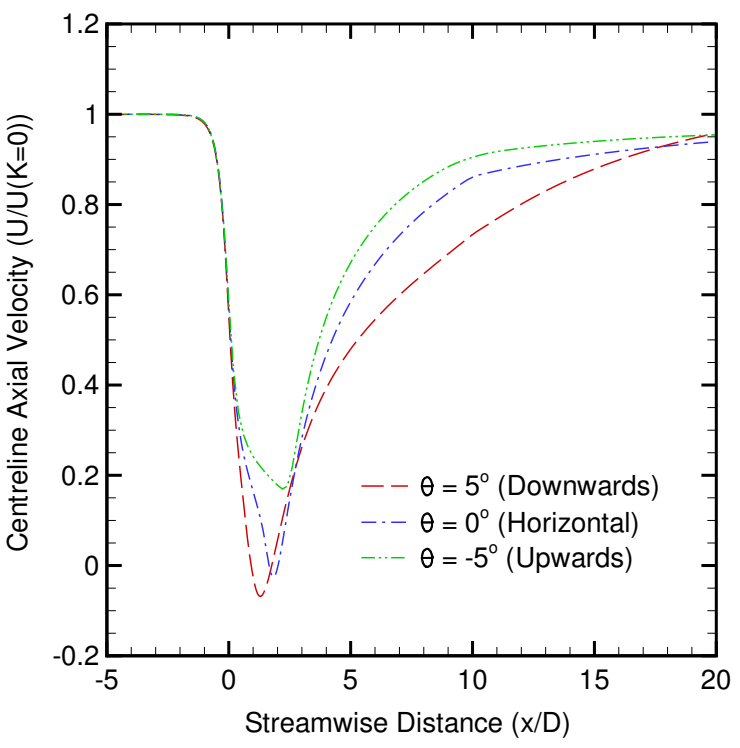

Figure 15: Centreline axial velocity along the local middepth of the channel for $K=5$, normalised by the centreline axial velocity along the mid-depth of the channel with no device present $(K=0)$, for the downwards facing $\left(\theta=5^{\circ}\right)$, horizontal $\left(\theta=0^{\circ}\right)$ and upwards facing $\left(\theta=-5^{\circ}\right)$ slopes.

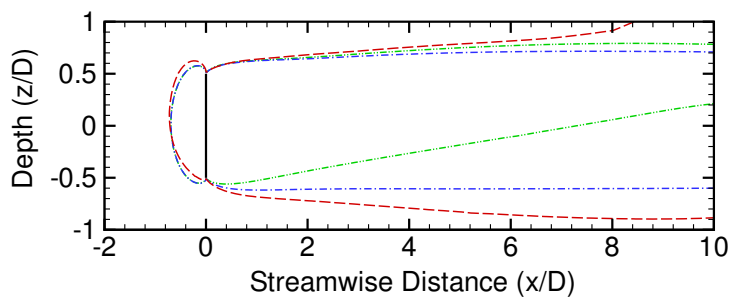

$$
\theta=5^{\circ} \text { (Downwards) } \theta=0^{\circ} \text { (Horizontal) } \theta=-5^{\circ} \text { (Upwards) }
$$

Figure 16: Location of the wake edge for $K=5$, here defined as the contour along which the axial velocity has reached $95 \%$ of the velocity with no device present $(K=0)$, for the downwards facing $\left(\theta=5^{\circ}\right)$, horizontal $\left(\theta=0^{\circ}\right)$ and upwards facing $\left(\theta=-5^{\circ}\right)$ slopes.

tually similar to the analytic model proposed by Houlsby et al. (2008) and Whelan et al. (2009). In their model, the downstream restriction of the bypass flow passage, occurred due to the drop in free surface height across the device. This drop in free surface height increased with increasing upstream Froude number and device thrust. The resulting decrease in downstream fluid depth lead to a similar increase in bypass flow velocity (as observed here).

Now consider the contours of static pressure co- 

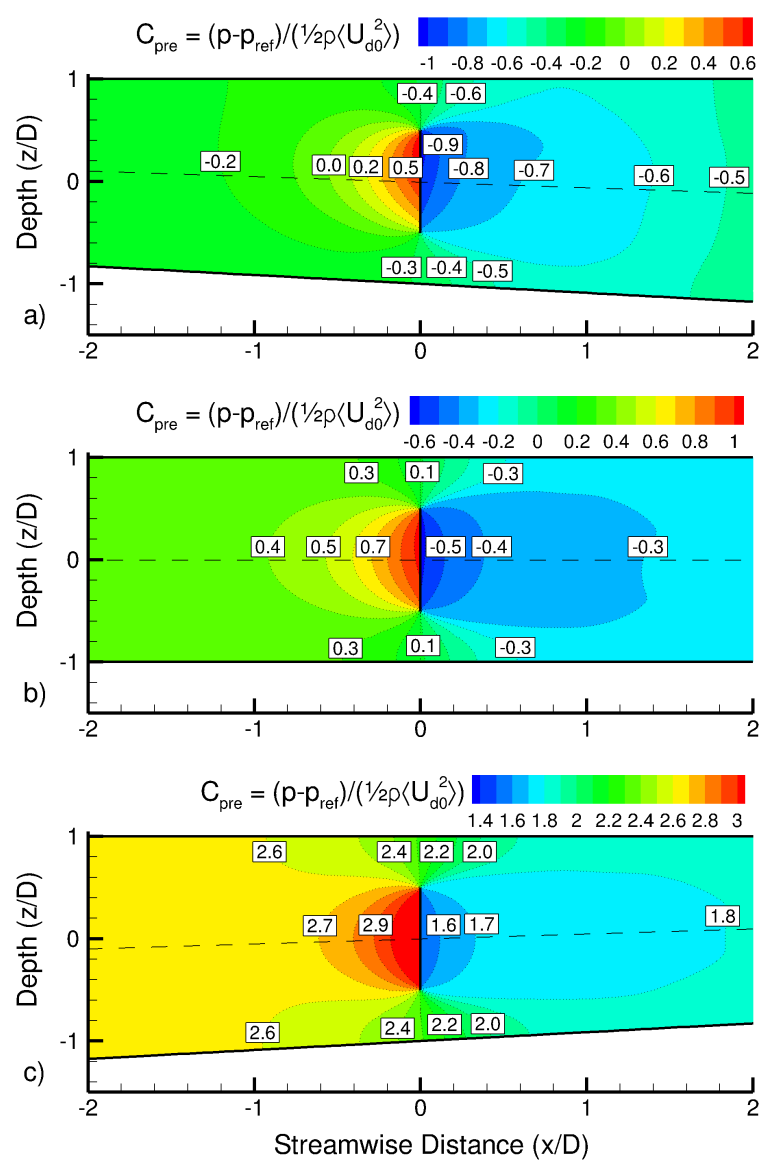

Figure 17: Static pressure coefficient contours for $K=5$ for (a) downwards facing slope $\left(\theta=5^{\circ}\right)$ (b) horizontal slope $(\theta=$ $\left.0^{\circ}\right)$ (c) upwards facing slope $\left(\theta=-5^{\circ}\right)$, for the high blockage case $(B=0.196)$. The dashed line indicates the local middepth of the fluid channel and the reference pressure $p_{\text {ref }}$ used in the definition of the static pressure coefficient $C_{\text {pre }}$, was taken at the computational domain outlet $(x / D=20)$.

efficient, shown in Fig. 17. The downwards facing slope experienced a drop in static pressure coefficient across the bypass flow passage of $\approx 0.3$, between $x / D=-2$ and 2. Whereas, the horizontal slope experienced a drop of $\approx 0.7$ and the upwards acing slope a drop of $\approx 0.8$. The increased static pressure coefficient drop across the bypass flow passage for the upwards facing slope was consistent with the increased bypass flow velocity, induced by the bed slope. As expected, the downwards facing slope did not experience such a large pressure coefficient drop, due to the reduced acceleration of the bypass flow.

The static pressure must equalise between the core and bypass flow, both downstream and up-

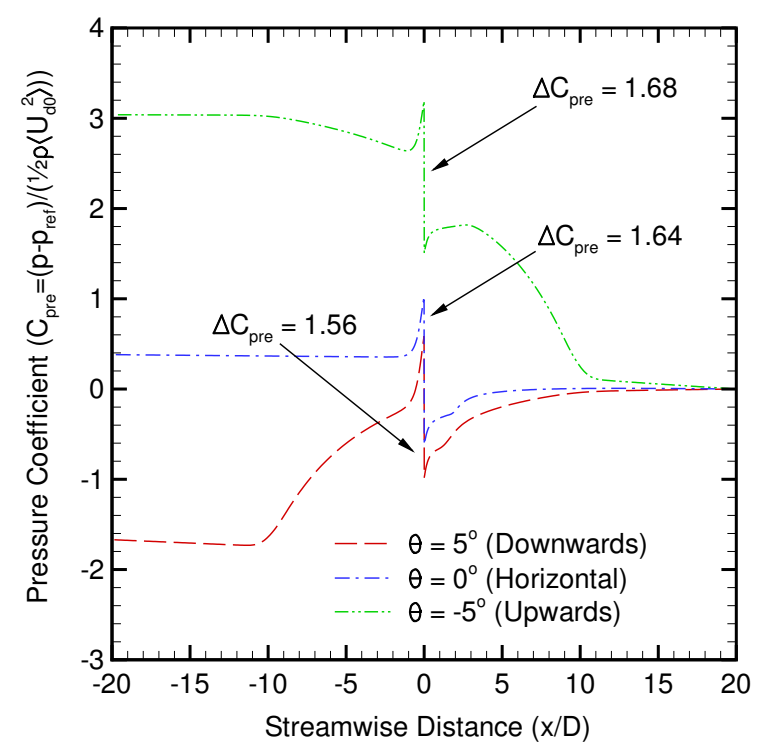

Figure 18: Static pressure coefficient $C_{\text {pre }}$ along the mid depth of the channel, for the downwards facing $\left(\theta=5^{\circ}\right)$, horizontal $\left(\theta=0^{\circ}\right)$ and upwards facing $\left(\theta=-5^{\circ}\right)$ slopes.

coefficient drop across the bypass flow was accompanied by an increased pressure coefficient drop across the core flow and hence across the disc. The pressure coefficient drop across the disc is difficult to accurately determine from the contours shown in Fig. 17. Instead, consider the pressure coefficient variation along the local mid depth of the channel (indicated by the dashed line in Fig. 17). As shown in Fig. 18, the upwards facing slope exhibited a greater pressure coefficient drop $\left(\Delta C_{\text {pre }}=1.69\right)$ across the disc, than the horizontal $\left(\Delta C_{\text {pre }}=1.65\right)$ and downwards facing slopes $\left(\Delta C_{\text {pre }}=1.61\right)$. This was consistent with the greater bypass flow acceleration and bypass flow pressure coefficient drop, experienced by the upwards facing slope. Note that the values for the drop in static pressure coefficient across the disc have been emphasised here, as they are difficult to read directly from the discontinuity in Fig. 18 with sufficient accuracy to discern a noticeable difference between the cases.

Now consider the variation in disc thrust and power coefficient, shown in Fig. 19. In this instance, an alternative power coefficient $\left(C_{p}^{\prime}\right)$ has been adopted as the ratio of power removed from the flow by the disc to the integral of the kinetic energy flux over the disc area with no device present, using equation 9. Likewise, an alternative thrust coefficient $\left(C_{T}^{\prime}\right)$ has been adopted using equation 
a) $\mathrm{B}=0.196$

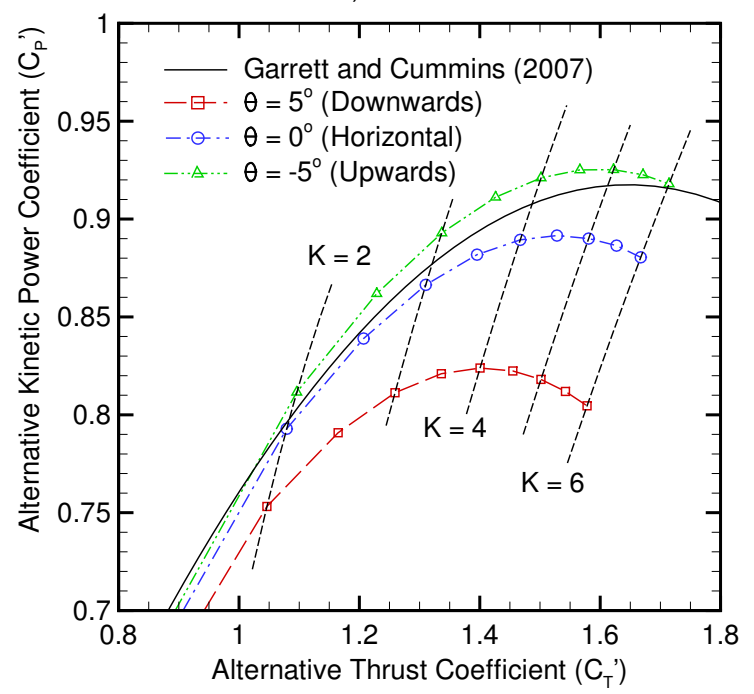

b) $B=0.065$

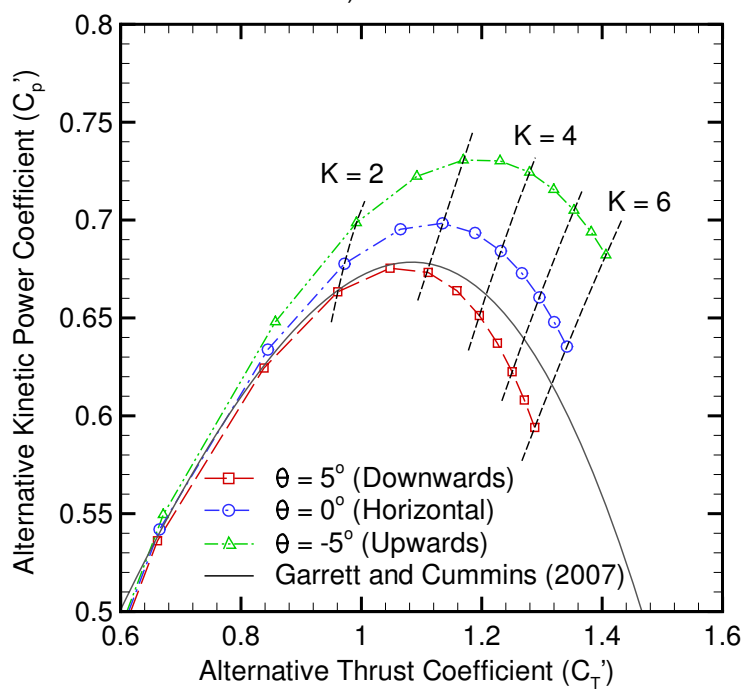

Figure 19: Kinetic power coefficient variation with thrust coefficient (based on the alternative normalisation) for the downwards facing $\left(\theta=5^{\circ}\right)$, horizontal $\left(\theta=0^{\circ}\right)$ and upwards facing slopes $\left(\theta=-5^{\circ}\right)$, for a) the high blockage $(B=0.196)$ and $\left.\mathrm{b}\right)$ the low blockage case $(B=0.065)$.

10 as the ratio of the thrust applied by the disc to

the flow to the integral of the dynamic pressure over the disc area, with no device present.

$$
\begin{aligned}
& C_{p}^{\prime}=\frac{\int_{A} \frac{1}{2} \rho K U_{d}^{3} d A}{\int_{A} \frac{1}{2} \rho U_{d 0}^{3} d A}=K \frac{\left\langle U_{d}^{3}\right\rangle}{\left\langle U_{d 0}^{3}\right\rangle} \\
& C_{T}^{\prime}=\frac{\int_{A} \frac{1}{2} \rho K U_{d}^{2} d A}{\int_{A} \frac{1}{2} \rho U_{d 0}^{2} d A}=K \frac{\left\langle U_{d}^{2}\right\rangle}{\left\langle U_{d 0}^{2}\right\rangle}
\end{aligned}
$$

As demonstrated in Fig. 19, for a given momentum loss factor, the upwards facing slope exhibited a greater alternative power and alternative thrust coefficient than the equivalent case on the horizontal and downwards facing slopes. This was consistent with the greater pressure coefficient drop across the core flow passage due to the downstream constriction of the flow passages, from the bed slope and free surface. Such trends were evident for both high and low blockage cases.

\section{Conclusions}

The bed slope has been shown to affect both the mean velocity profile at the disc plane and the flow passage expansion downstream of the disc, at high and low blockage ratios. Whilst an upwards facing slope achieves higher pressure coefficient drop due to downstream flow constriction, the highly sheared velocity profile exhibited on downwards facing slopes leads to greater potential power delivery. However device operation on an upwards facing slope may be more desirable (for a given mass flow rate and local depth) as the mean velocity profile across the projected area of the device is more uniform, leading to reduced thrust variation. For a real device, the closer to uniform velocity profile is likely to result in lower cyclical blade load fluctuations and thus fatigue damage contribution during uphill flows, albeit at lower power delivery than can be achieved for downhill flows if such velocity shear can be efficiently converted into realizable power.

\section{Acknowledgement}

The author would like to thank EON and EPSRC for funding the CASE-Studentship for the project and the Advanced Research Computing (ARC) facility at the University of Oxford, where the computations where performed.

\section{References}

Apsley, D. D., Leschziner, M. A., 2000. Advanced turbulence modelling of separated flow in a diffuser. Flow, Turbulence and Combustion 63, 81-112. 
Buice, C. U., Eaton, J. K., 1997. Experimental investgation of flow through an asymmetric plane diffuser. Tech. Rep. TSD-107, Department of Mechanical Engineering, Stanford University.

Cebeci, T., Bradshaw, P., 1977. Momentum Transfer in Boundary Layers. Hemisphere Publishing Corporation, New York, USA.

Consul, C., Willden, R. H. J., McIntosh, S. C., 2013. Blockage effects on the hydrodynamic performance of a marine 905 cross flow turbine. Phil. Trans. R. Soc. A. 371.

Draper, S., Borthwick, A., Houlsby, G., 2013. Energy potential of a tidal fence deployed near a coastal headland. Philosophical Transactions of the Royal Society of London A: Mathematical, Physical and Engineering Sciences 910 371 (1985).

Edmunds, M., Malki, R., Williams, A., Masters, I., Croft, T., 2014. Aspects of tidal stream turbine modelling in the natural environment using a coupled BEMCFD model. International Journal of Marine Energy 7, 20-42.

E.ON, October 2014. Private Communication.

Evans, P., Lazarus, E., Mason-Jones, A., ODoherty, D., ODoherty, T., September 2015. Wake characteristics of a natural submerged pinnacle and implications for tidal stream turbine installations. In: Proceedings of the 11th 920 European Wave and Tidal Energy Conference. Nantes, France.

Gant, S., Stallard, T., July 2008. Modelling a tidal turbine in unsteady flow. In: International Offshore and Polar Engineering Conference. Vancouver, Canada, pp. 473-479.

Garrett, C., Cummins, P., 2007. The efficiency of a turbine in a tidal channel. Journal of Fluid Mechanics 588, 243-251.

Hellsten, A., Rautaheimo, P., June 1999. Proceedings of the 8th ERCOFTAC/IAHR/COST workshop on refined turbulence modelling. Helsinki Univiersity of Technology.

Houlsby, G. T., Draper, S., Oldfield, M. L. G., 2008. Application of linear momentum actuator disc theory to open channel flow. Tech. Rep. OUEL 2296/08, Department of Engineering Science, Univerity of Oxford.

Kaltenbach, H. J., Fatica, M., Mittal, R., Lund, T. S., Moin, P., 1999. Study of flow in a plane asymmetric diffuser using large-eddy simulation. Journal of Fluid Mechanics 390, 151-185.

Launder, B. E., Sharma, B. I., November 1974. Application of the energy-dissipation model of turbulence to the calculation of flow near a spinning disc. Letters in Heat and Mass Transfer 1 (2), 131-138.

Masters, I., Malki, R., Williams, A., Croft, T., 2013. The influence of flow acceleration on tidal stream wake dynamics: A numerical study using a coupled BEM-CFD model. Applied Mathematical Modelling 37, 7905-7918.

Menter, F. R., 1994. Two-equation eddy-viscosity turbulence models for engineering applications. AIAA Journal 32(8), 1598-1605.

885 Menter, F. R., Kuntz, M., Langtry, R., 2003. Ten years of industrial experience with the SST turbulence model. Turbulence, Heat and Mass Transfer 4, 625-632.

Nishino, T., Willden, R. H. J., 2012. Effects of 3-D channel blockage and turbulent wake mixing on the limit of power extraction by tidal turbines. International Journal of Heat and Fluid Flow 37, 123-135.

Obi, S., Aoki, K., Masuda, S., August 1993. Experimental and compuational study of turbulent seperating flow in an asymetric plane diffuser. In: Ninth Symposium on Turbulent Shear Flows. Kyoto, Japan.

Patankar, S. V., 1980. Numerical Heat Transfer and Fluid
Flow. Hemisphere Publishing Corporation, Washington DC USA.

Pope, S. B., October 2000. Turbulent Flows. Cambridge University Press, The Edinburgh Building, Cambridge, UK.

Rhie, C. M., Chow, W. L., 1983. Numerical study of turbulent flow past an aerofoil with trailing edge seperation. AIAA journal 21, 1525-1532.

Soto, K., Escauriaza, C., September 2015. Numerical simulations of marine hydrokinetic devices in complex bathymetries. In: Proceedings of the 11th European Wave and Tidal Energy Conference. Nantes, France.

Tabor, G. R., Baba-Ahmadi, M. H., 2010. Inlet conditions for large eddy simulation: A review. Computers and Fluids $39,553-567$.

Whelan, J. I., Graham, J. M. R., Peiró, J., 2009. A freesurface and blockage correction for tidal turbines. Journal of Fluid Mechanics 624, $281-291$.

Wilcox, D. C., 1988. Re-assessment of the scale-determining equation for advanced turbulence models. AIAA Journal 26(11), 1299-1310.

Wilcox, K., McLeod, I., Gerber, A., Jeans, T., Culina, J., September 2015. Validation of high-fidelity CFD simulation of the unsteady turbulent flow in Minas Passage. In: Proceedings of the 11th European Wave and Tidal Energy Conference. Nantes, France.

Zangiabadi, E., Edmunds, M., Fairley, I., Togneri, M., Williams, A., Masters, I., Croft, N., 2015. Computational fluid dynamics and visualisation of coastal flows in tidal channels supporting ocean energy development. Energies 8, 5997-6012. 